\title{
Archipel
}

ARCHIPEL Études interdisciplinaires sur le monde insulindien

$89 \mid 2015$

Varia

\section{Maps of Malaya and Borneo: Discovery, Statehood and Progress. The Collections of H.R.H. Sultan Sharafudin Idris Shah and Dato' Richard Curtis}

Daniel Perret

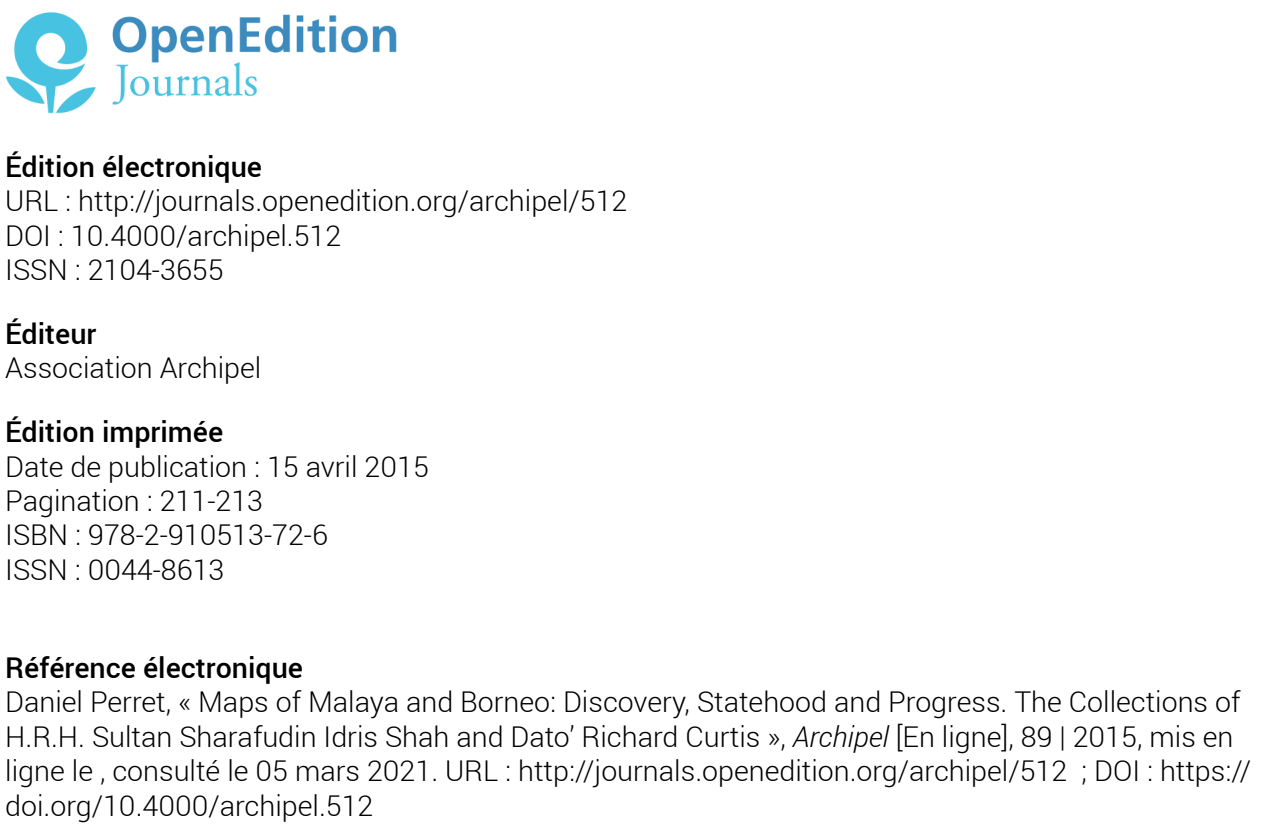

Ce document a été généré automatiquement le 5 mars 2021

Association Archipel 


\title{
Maps of Malaya and Borneo: Discovery, Statehood and Progress. The Collections of H.R.H. Sultan Sharafudin Idris Shah and Dato' Richard Curtis
}

\author{
Daniel Perret
}

\section{RÉFÉRENCE}

Frédéric DURAND in collaboration with Richard CURTIS, Maps of Malaya and Borneo: Discovery, Statehood and Progress. The Collections of H.R.H. Sultan Sharafudin Idris Shah and Dato' Richard Curtis. Kuala Lumpur: Éditions Didier Millet / Jugra Publication, 2013, 264 p., Liste des cartes, glossaire, bibliographie, index, 27 x 28,6 cm (ISBN:

978-983-44773-7-0), 32 x 34 cm. ISBN: 978-967-10617-3-2.

1 C'est probablement une coïncidence qui a fait que Maps of Malaya and Borneo a été publié l'année même du cinquantenaire de la formation de la Fédération de Malaysia, concrétisée par le rattachement de Sarawak et de Sabah à la Fédération de Malaya en septembre 1963. Deux ans plus tôt, Paul Wheatley avait fait paraître à Kuala Lumpur The Golden Khersonese (University of Malaya Press, 1961), étude majeure de géographie historique sur la péninsule malaise ancienne, qui fait encore référence.

2 Si son propos est différent, à sa manière,Maps of Malaya and Borneo fait également figure d'ouvrage pionnier. C'est en effet, semble-t-il, la première fois qu'un ouvrage est consacré spécifiquement à des collections de cartes relatives à la Malaisie. Les deux corpus présentés ici, initiés à la fin des années 1970, appartiennent respectivement à l'actuel sultan de l'État de Selangor et à l'un des hommes d'affaires les plus en vue de l'État de Sarawak, fils d'un haut fonctionnaire de l'administration coloniale britannique 
en Malaisie. Comportant 161 cartes issues de ces collections, qui couvrent la période 1513-1969, l'ouvrage est en réalité plus riche que ce qu'annonce le titre, puisqu'il est aussi illustré de cinquante cartes, conservées dans une vingtaine de fonds essentiellement publics, malaysiens et étrangers.

3 À la suite d'une introduction liminaire par Frédéric Durand (p. 14-21), l'ouvrage est agencé en deux parties principales : une introduction historique et une description des cartes appartenant aux deux collectionneurs. L'introduction de Frédéric Durand (p. 24-69) comporte treize courts chapitres retraçant les grandes étapes de la cartographie de la région.

4 La difficulté avec ce genre de corpus, c'est comment lui donner un sens, dans la mesure où les collectionneurs ont certainement enrichi leurs collections au gré des opportunités d'achat plutôt qu'en fonction d'une thématique particulière. La partie descriptive et commentée des collections (p. 71-239) est ainsi divisée en huit thèmes : premières cartes imprimées occidentales (14 cartes), influences néerlandaises, françaises et anglaises initiales (22 cartes), approfondissement de la connaissance cartographique ( 13 cartes), chartes nautiques ( 35 cartes), cartes de l'intérieur (30 cartes), cartes d'atlas et d'encyclopédies (14 cartes), cartes montrant l'économie, la culture et les communications (17 cartes), cartes urbaines (16 cartes).

5 Peut-être par souci esthétique ou parce que l'histoire en est considérée comme bien balisée, la partie introductive du volume ne comporte pas d'appareil critique. Si l'on peut comprendre que cet ouvrage s'adresse d'abord au grand public, on pourra regretter que ce choix éditorial laisse aux seuls spécialistes d'histoire de la cartographie la possibilité d'apprécier les approches et idées nouvelles livrées par Frédéric Durand, l'un des rares experts français de l'histoire de la cartographie de l'Asie du Sud-Est.

6 Sans surprise, les collections montrent une histoire de la cartographie largement dominée par les Occidentaux. On notera en particulier les contributions de fonds français et les passages concernant les cartographes français (p. 47-8, 50). Curieusement, la cartographie exacte des contours de Bornéo (années 1880) semble achevée avant celle de la péninsule (début $\mathrm{xx}^{\mathrm{e}} \mathrm{s}$.) (p. 16-17).

7 Frédéric Durand signale utilement que le nom de "Melacha » pour désigner l'actuelle Melaka apparaît sur une carte occidentale dès 1459 (p. 27), quelques décennies après les cartes chinoises élaborées lors des expéditions maritimes Ming (1405-1433) (p. 28). L'existence d'une cartographie locale (javanaise et malaise ?) dès le début du $\mathrm{xvI}^{\mathrm{e}}$ siècle, dont malheureusement pratiquement rien ne subsiste, est également rappelée (p. 32, 52).

8 Plusieurs documents inédits sont à signaler, en particulier une carte partielle de Sarawak des années 1890 (doc. no 97 p. 175) et un plan de Victoria (Labuan), c. 1911 (doc. $n^{\circ} 158$, p. 236). Certains documents auraient mérité un plus grand format, voire une double page, telles les exceptionnelles cartes sud-est asiatiques (p. 57, 59).

9 Il semble assez étonnant que les deux collections ne comprennent que trois cartes de Kuala Lumpur, la plus ancienne étant datée de 1925, alors que la ville compte déjà plus de 40000 habitants à la fin du siècle précédent. Par ailleurs, il ne semble pas exister de cartes japonaises de la région avant les années 40.

Rejoignant l'ouvrage de Paul Wheatley mentionné plus haut, les cartes les plus anciennes soulèvent toujours de nombreux problèmes de localisation toponymique. Pour ne citer qu'un exemple, le Barussae insulae de la carte de Ptolémée, placé à 
proximité de la péninsule, qui estgénéralementidentifié avec Barus sur la côte ouest de Sumatra Nord (p. 25). Or, une autre interprétation est possible : il pourrait s'agir de l'archipel des Maldives, dans la mesure où l'une des îles de l'atoll de Malé s'appelle Baros.

11 Nous n'avons relevé qu'une erreur: Sabah et Sarawak intègrent la $F$ édération en 1963 et non pas 1964 (p. 19).

12 Cet ouvrage donne indéniablement envie d'en savoir plus: ces deux collections représentent quel pourcentage des cartes connues? Quels sont les grands fonds publics de cartes sur la question? Pour les plus anciennes, combien d'exemplaires en sont recensés aujourd'hui?

13 Il inspire également deux réflexions plus générales. Si la question de la cartographie des terres émergées du globe semble aujourd'hui réglée, la disparition d'un avion de la Malaysian Airlines en mars 2014 amène à constater que c'est loin d'être le cas pour la cartographie des fonds marins, quelque trois siècles après l'apparition des premières indications de profondeurs bathymétriques sur les cartes de la région ( $c f$. carte de Pierre vander Aa, 1719, p. 108). Par ailleurs, c'est paradoxalement au moment où la sortie de cet ouvrage va immanquablement sensibiliser nombre de Malaysiens à un aspect jusque-là négligé de leur patrimoine, qu'on est en droit de s'interroger sur l'avenir de l'édition de la carte imprimée seule, une tradition qui naît au milieu du XVI siècle.

On ne peut que saluer cette initative des collectionneurs et de Frédéric Durand, qui a su mettre en valeur avec clarté et concision la richesse du corpus dans un livre splendidement illustré, avec une mise en page très soignée. 